\title{
Variable Structure Control of Shape Memory Alloy Actuators
}

\author{
Danny Grant and Vincent Hayward
}

A novel shape memory alloy (SMA) actuator consisting of a number of thin NiTi fibers woven in a counter rotating helical pattern around supporting disks is first described. This structure accomplishes a highly efficient transformation between force and displacement overcoming the main mechanical drawback of shape memory alloys, that being limited strain.

Time domain open loop experiments were then conducted to determine the intrinsic properties of the actuator. From these experiments and from the knowledge of the underlying physics of SMAs, a multi-term model, including linear and nonlinear elements, was proposed. After further investigation and simulation, it was found that most of these complexities did not need to be considered in order to explain the reported results, and that the model could be reduced to that of a single integrator.

A variable structure controller was then applied to a pair of antagonist actuators. The feedback switches between the two actuators according to the sign of the displacement error. A further improvement was added to compensate for known gross nonlinearities by modulating the current magnitude in a discrete manner as a function of the state space position. It was therefore possible to realize smooth and robust control with very little cost in complexity.

\section{Introduction}

With the continued miniaturization of robotic systems comes the need for powerful, compact, lightweight actuators. Conventional techniques such as electric, hydraulic, and pneumatic actuators, suffer from a drastic reduction of the amount of power they can deliver as they scale down in size and weight [8]. To overcome this limitation, different actuator technologies have been investigated, in particular shape memory alloys (SMA). Shape memory alloys have a high strength-to-weight ratio, which makes them ideal for miniature applications. A SMA fiber can achieve a maximum pulling force of $180 \mathrm{MPa}$. Comparing this to an electro-magnetic actuator, which can only achieve .002 $\mathrm{MPa}$ [7], this represents an almost $10^{5}$ increase in strength per cross sectional area. Thin fibers of shape memory alloy can accomplish actuation by being pretreated to contract upon heating. The contraction is a result of the fiber undergoing a phase transition between its Martensitic and Austenitic phases. When in the cool phase (Martensitic) the alloy is malleable and can easily be

The authors are with the Centre for Intelligent Machines, McGill University,Montreal,Quebec, Canada,grant@cim.mcgill.ca,hayward@cim.mcgill.ca. This article is an updated version of a paper that was presented at the 1995 IEEE International Conference on Robotics and Automation held in Nagoya, Japan. deformed by applying external stress. The original pretrained shape can then be recovered by simply heating the fiber above its phase transition temperature. Since the alloy is resistive it can easily be heated electrically.

Along with SMA's high strength-to-weight ratio come several limitations. SMAs possess highly nonlinear properties [1], such as a complicated hysteresis loop in the transition temperature relationship. They can, however, still be controlled through the use of feedback and other control techniques, and a variable structure controller will be applied in the second part of this article. The main physical limitation that needs to be overcome is the absolute percent strain. SMAs can achieve a practical strain of 5 percent.

Many of the designs of actuators using shape memory alloys depend on mechanically amplifying the displacement either through the use of long straight fibers $[6,10,13]$, or through the use of coils $[7,8,10]$. The proposed actuator achieves mechanical motion amplification that is more compact than a long straight length of fiber, and more efficient than using coils.

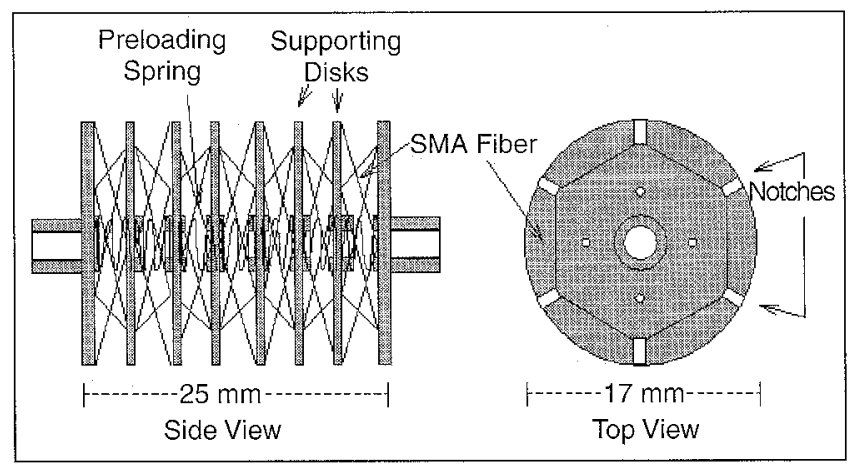

Fig. 1. Shape memory alloy actuator.

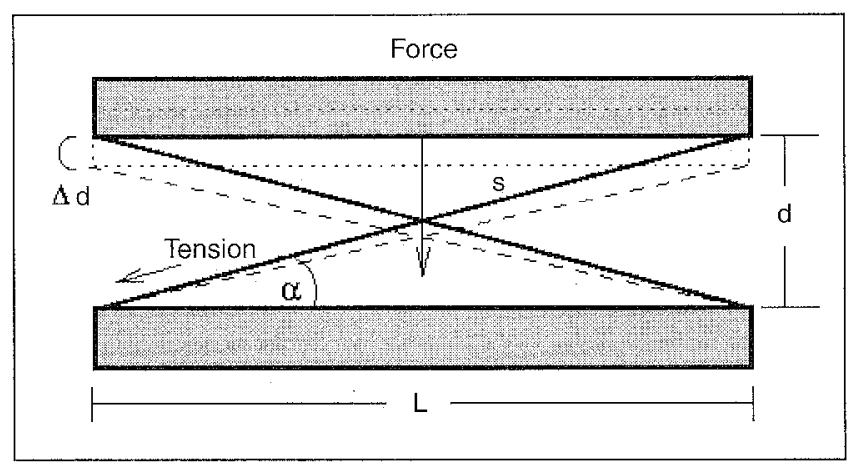

Fig. 2. Simplified case: two beams with two fibers. 


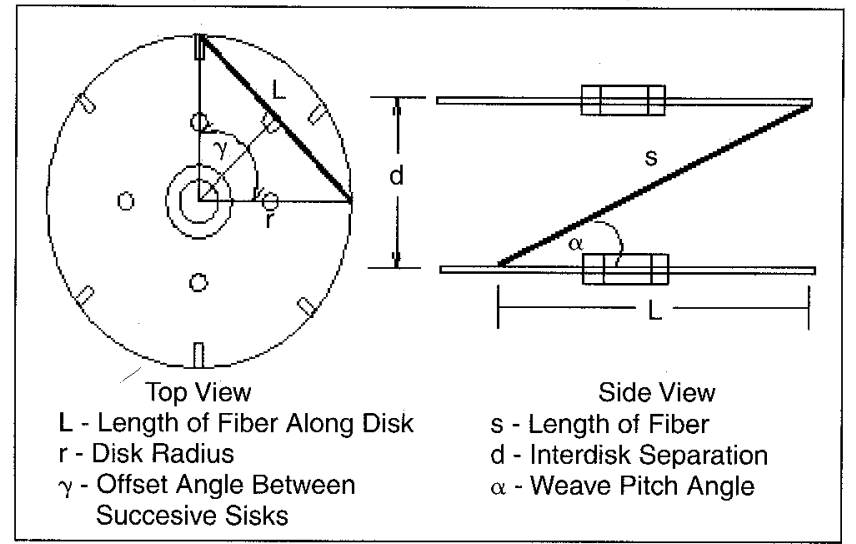

Fig. 3. Variables involved.

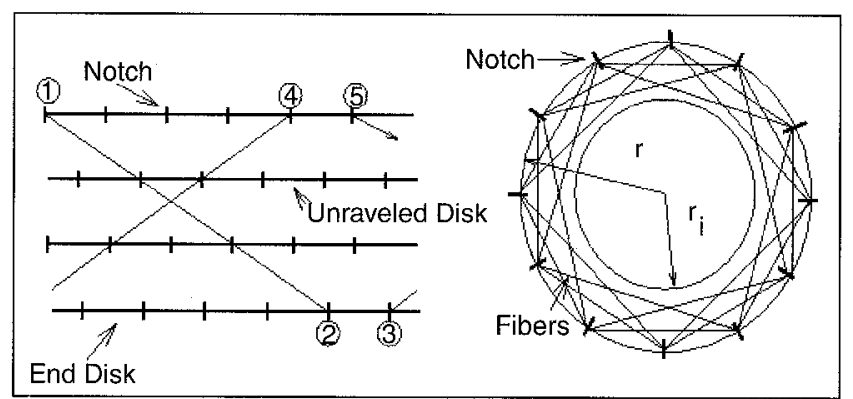

Fig. 4. Left: Unravelled fiber weave. Right: Axial view of completed weave.

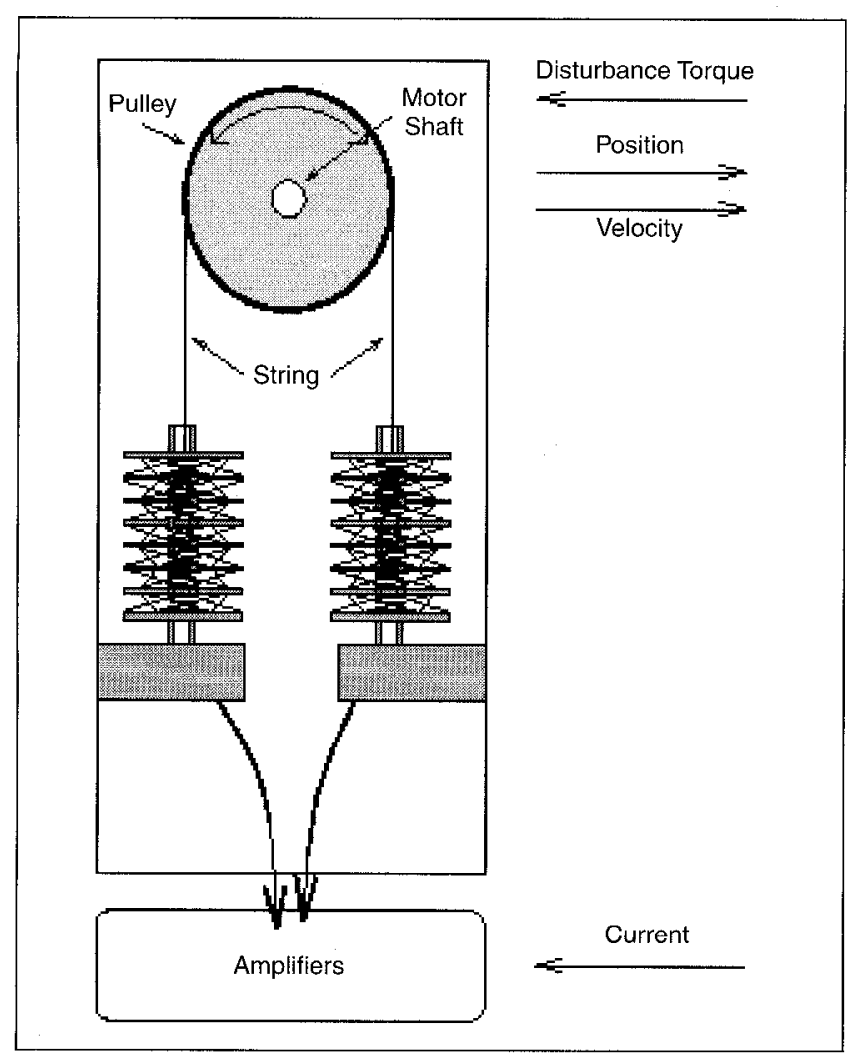

Fig. 5. Top view of testbed.

\section{Shape Memory Alloy Actuator}

The actuator, shown in Fig. 1, consists of 12 thin NiTi fibers woven in a counter rotating helical pattern around supporting disks. The disks are separated by preloading springs that keep the fibers under tension when relaxed. When the fibers are heated, they contract pulling the disks together.

The weave pattern of the fibers accomplishes a displacement amplification. Essentially the abundant force of the alloy is being traded off for a displacement gain. This transformation between force and displacement is highly efficient since the only loss in work is due to the slight bending of the fibers. Unlike shape memory alloy coils, the entire cross section of the fiber in the weave is performing work in the contraction. Coils suffer from the debilitating drawback of requiring a larger diameter than necessary. This is especially negative, since the response time is directly related to fiber diameter. The response of the actuator is limited by the cooling rate of the NiTi fibers, which directly depends on the fiber's surface area to volume ratio. The higher this ratio, the more rapidly the fiber will cool. A great deal of the material is wasted in SMA coils since, during the shape memory effect, only the skin of the coil is actually contracting at the maximum amount. The internal diameter of the coil acts both as a heat sink and as a source of opposing force to the desired motion.

The weave pattern also results in an ideal "tensegrity" structure [3], with all compression members being passive and all tension members active, resulting in an optimal use of the material.

\section{Simplified Case}

The kinematic amplification can best be seen by considering the simplified case consisting of two beams and two fibers as shown in Fig. 2.

As the two fibers contract, the two beams are pulled togetl er. The displacement gain, $\Delta d / \Delta s$, is defined as the change in str.ke along the separating distance, divided by the change in the fiber length. Since ideally the motion is constrained along $d$ the instantaneous displacement gain is given by:

$$
\frac{\delta d}{\delta s}=\frac{s}{\sqrt{s^{2}-L^{2}}}=\frac{1}{\sqrt{1-\cos \alpha^{2}}}=\frac{1}{\sin \alpha}
$$

The displacement gain is inversely proportional to the sine of the weave pitch. As the disks get closer together, the displacement gain dramatically increases, asymptotically approaching infinity.

\section{SMA Actuator}

The weave pattern of the SMA actuator achieves a kinematic amplification for each cell of the actuator. All the radial components of the tension forces of the twelve fibers cancel, leaving only a common axial stress force component. In this manner the displacement gain overcomes the main mechanical drawback of the SMA, while the force attenuation is compensated by using several fibers in parallel. The actuator is no longer limited to the absolute percent strain of the fiber. The displacement gain also allows the fiber to operate at reduced percent absolute strain, and since the cycle lifetime of the SMA fibers increases dramatically when they operate at a lower than absolute strain, the cycle lifetime can also be increased. To obtain a reasonable response, 100 $\mu \mathrm{m}$ diameter SMA fibers were chosen for the actuator prototype. 
Twelve $100 \mu \mathrm{m}$ fibers acting in parallel, allow rapid cooling in ambient air without compromising strength.

\section{Design Parameters}

The prototype represents only one configuration of the SMA actuator's parameters [5]. The supporting disk size and spacing, the number of fibers, and the displacement gain are all adjustable parameters. Fig. 3 will define the variables involved, highlighting only one of the fibers in a single actuator cell.

All the parameters are interrelated leading to an engineering problem for which an acceptable tradeoff must be found within set constraints. Equation (1) shows that the displacement gain is inversely proportional to the sine of the weave pitch. The weave pitch in turn is dependent on the fiber weave pattern and the radius and spacing of the supporting disks. The weave pattern is determined by the number of notches around the disk, and the relative alignment of successive disks.

The offset angle, $\gamma$, is the angle between notches of successive disks in the actuator. For the actuator prototype in Fig. 1, eight disks were chosen with six notches spaced 60 degrees apart. The prototype actuator was constructed by aligning the disks vertically so that each successive disk was offset by 30 degrees. The weave pattern was obtained by threading a single fiber along the notches of the eight disks. Adjacent disks were connected by the fiber through notches that were separated by an offset angle of 90 degrees. The two end disks are woven along successive notches as shown in Figure 4. To get a better idea of how the fibers are woven, imagine the support disks of the actuator rolled out so that they are flat. The left of Fig. 4 shows a four disk actuator with the disks unravelled. The fiber weave would begin at an end disk and pass through the successive points one through five.

The fiber would then continue going back and forth between the two end disks until it arrived back at its starting position. In this manner it is possible to manually connect many fibers in parallel, simply and securely using a small jig in a manner of minutes. After the weave was completed the two ends of the fiber were merely tied in a knot. This also provided a secure mechanical connection as most of the stress on the fiber occurs at the notches. The final result is 12 fibers woven in counter helical rotations such that all radial forces cancel out upon contraction.

\section{Displacement Gain}

The displacement gain can be increased by increasing the offset angle, $\gamma$, or by decreasing the inter-disk distance $d$. There are of course limits on both of these parameters. As the offset angle approaches 180 degrees, the fibers approach the center of the disk. This causes the structure to become more unstable and reduces the available space in the center for the placement of the springs and/or a position sensor. The radius of the inner bounding cylinder, shown on the right of Fig. 4 can be found by trigonometry to be $r_{i}=r * \cos \gamma$ where $r$ is the disk radius and $\gamma$ is the offset angle.

Decreasing the distance in between the disks dramatically increases the displacement gain but limits the amount of stroke per cell. If the disks begin very close together they can only move a. small distance before they come in contact with one another.

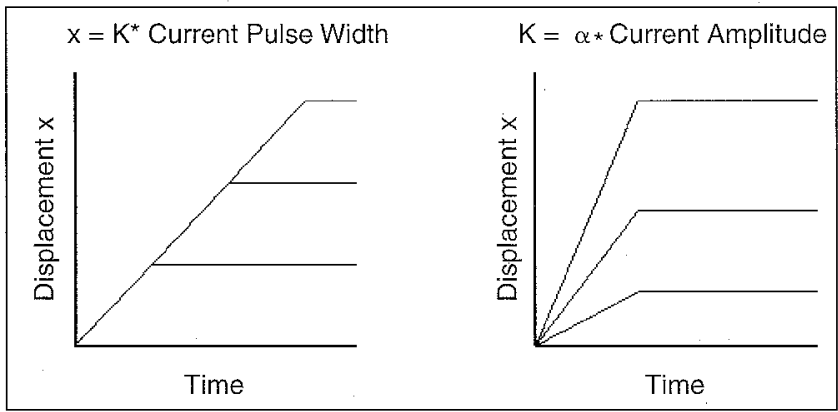

Fig. 7. Curves of an ideal integrator.

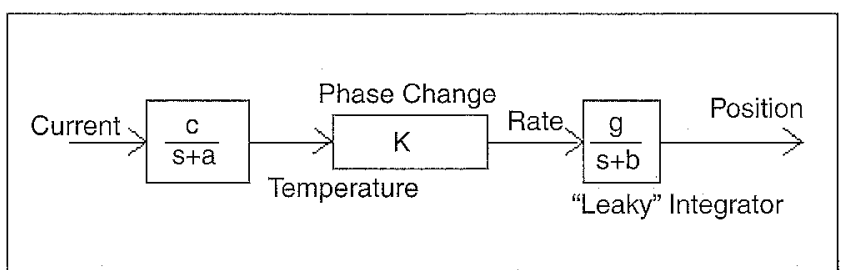

Fig. 8. Simplified model.

The force generated by the actuator can be adjusted by choosing the number and size of fibers used in the weave. Obviously the more fibers that are acting in parallel the larger the force generated. Again there is a limitation here on the number of fibers that can be used. As the number of fibers increases so does the fiber interference in the weave. This, however, is not a major problem due to the flexible nature of the fiber. Fibers with a larger diameter can be chosen, but at the expense of response, as cooling times will increase. The actuator is also modular in the sense that any number of cells can be cascaded together.

\section{Controller Design}

A considerable amount of work has been concerned with the modeling of shape memory alloy actuators $[2,9,12]$, while relatively less attention has been paid to the design of feedback control laws. Many feedback control techniques reported in the literature applied to shape memory actuators are in fact linear compensators such as P, PD or PID controllers $[12,13]$, or close cousins. 


\section{IEEE Systems and Control Maganize, Vol. 17, No. 3, pp. 80-88, 1997}

The dynamics of shape memory actuators are predominantly nonlinear since the energy conversion principle, from heat to mechanical, relies on exploiting phase transitions in a metal. This creates at least significant hysteresis in addition to many other nonlinear effects with or without memory. In addition, the detailed properties of the dynamics of shape memory alloys vary widely with their metallurgy content, fabrication process, training techniques [7], aging, and ambient conditions; thus a great deal of parametric uncertainty is involved. Moreover, most of the detailed descriptions of their underlying physics are often not very useful for controller design, and in the case of linear controllers one usually ends up with an overly conservative design.

There are several nonlinear control techniques available; however, due to the large uncertainty in plant parameters, several

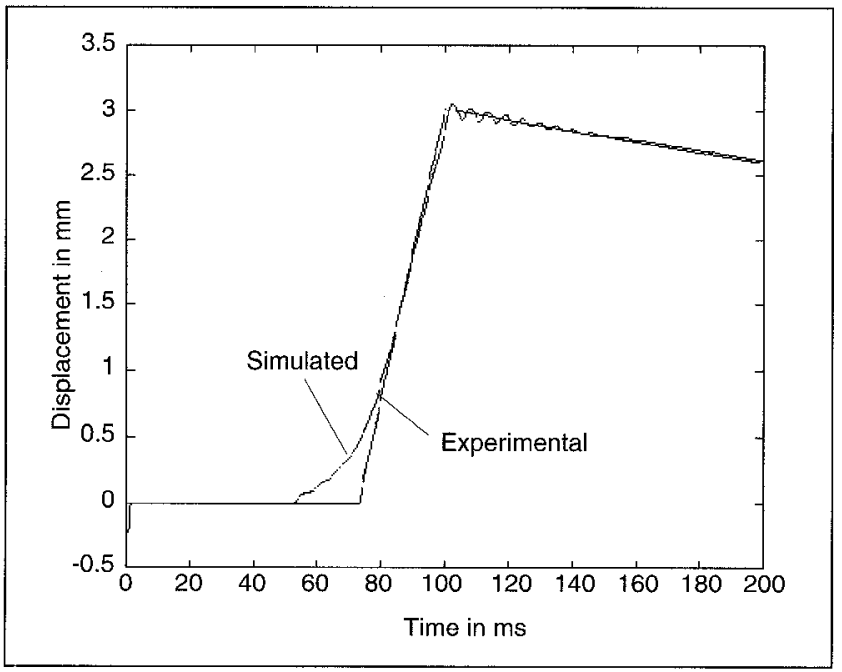

Fig. 9. Model results.

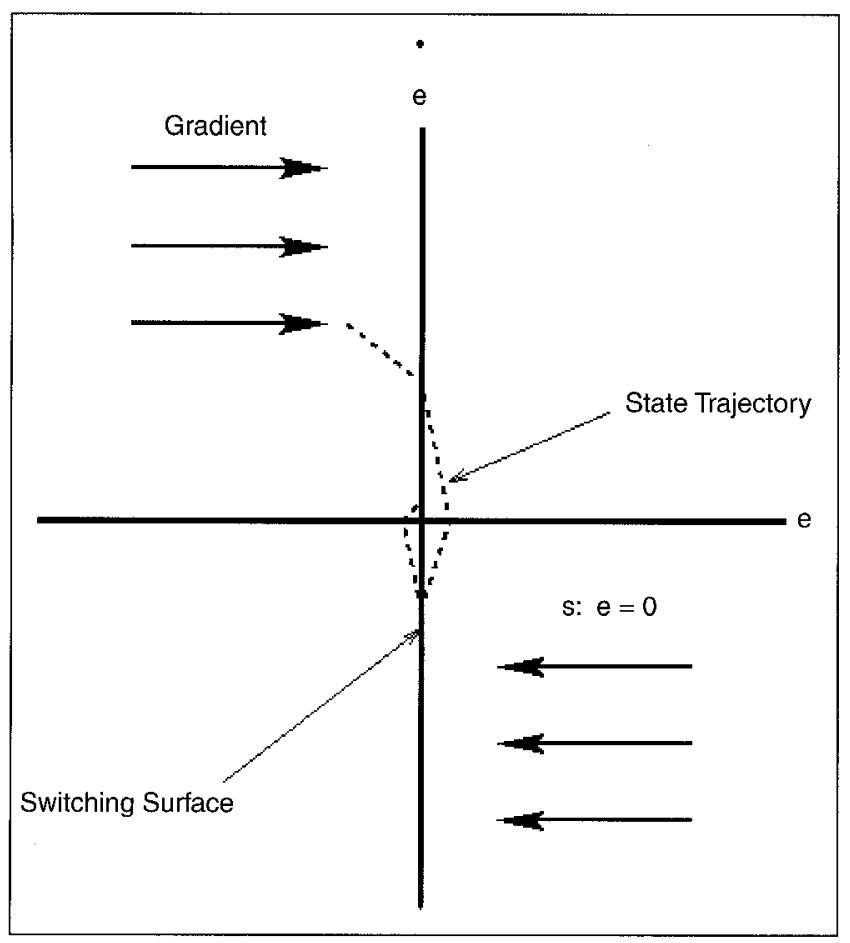

Fig. 10. Phase plot. were immediately discarded, such as those relying on precise nonlinear identification, adaption, and feedback linearization. Using describing functions was also ruled out as this would again result in an overly conservative controller. This naturally led to the use of robust variable structure control.

\section{Motivation for Variable Structure Control}

The overwhelming advantage of variable structure control is that relatively few parameters representing the knowledge of the physical properties of the plant need to be known, since only inequality conditions need to be satisfied in the design [14]. It is also well known that variable structure control is quite insensitive to plant parameter variations since the resulting trajectory resembles a time near-optimal switching curve.

It is often stated that a disadvantage of variable structure control is the discontinuous nature of the control signal, which may cause problems in actuators in terms of ringing, excessive dissipation, and excitation of unwanted dynamics in the plant being driven by these actuators. These problems sometimes can be solved by the introduction of smooth switching laws while retaining some of the advantages of the technique. Another approach is the introduction of boundary layers in the vicinity of the so-called sliding surfaces when the nature of the plant precludes the switching frequency to approach infinity; see, for example, [11].

For some actuation techniques, switching is not a problem and clearly shape memory alloy actuation is one of them: the mechanical energy is derived from heat which makes the actuators naturally low pass, and thus the higher order dynamics are undisturbed by step or impulse inputs. Moreover, the robustness properties of variable structure control combined with the modeling difficulties of shape memory alloy actuators creates considerable incentive to apply the former to the latter.

There is also yet another exceedingly practical reason that makes this combination attractive. No amplifiers, valves, or other continuous energy throttling mechanisms are needed to realize the control, only switches. As previously mentioned, one great attraction of shape memory alloy actuators is the possibility for miniaturization. With variable structure control, the energy throttling device can be as simple as a single FET switching current on and off from a power bus, thereby opening a path toward a mechatronic-type high degree of integration with actuation, sensing, control and energy throttling including in a single unit.

\section{Experimental Setup}

The fibers used in the actuator only exhibit the one-way shape memory effect. For this reason it is necessary to force bias individual actuators so that they will return to their original length when cooled. This can be accomplished easily by using biasing springs or by using actuators in an antagonistic fashion. Shape memory alloys are especially suited to antagonistic arrangements since the force required to deform the alloy is much less than the force generated by the phase transformation. Using the actuators in an antagonistic fashion also results in improved system response. The response time of the actuator system will depend strongly on heat activation, which can be tuned according to the input current amplitude. For these reasons open-loop experiments were performed using two actuators in an antagonistic fashion as shown in Fig. 5. 


\section{Open-Loop Response}

The time domain open-loop response to a step of input current applied to the actuator can be seen in Fig. 6. The left side of Fig. 6 shows the results of heating one of the actuators with a $50 \mathrm{~ms}$ pulse (starting at $50 \mathrm{~ms}$ ) while varying the current amplitude by $0.3 \mathrm{~A}$ steps from $4.2 \mathrm{~A}$ to $7.8 \mathrm{~A}$. Note that the 12 fibers are electrically connected in parallel; hence the current in each fiber is divided by a factor of 12 .

The right side of Fig. 6 shows the results of heating the actuator with a constant current of $6.3 \mathrm{~A}$ and varying the duration of the pulse between $20 \mathrm{~ms}$ and $65 \mathrm{~ms}$ in $5 \mathrm{~ms}$ steps.

\section{Coarse Modeling from the Open-Loop Response}

Adopting a black box modeling approach, the open loop step response of the actuator reveals that although the phase transformation involves highly nonlinear properties, a markedly linear relationship between heating time and percent strain can be observed in a large portion of the graph. A coarse model can be written as:

$$
\text { Displacement }=K * t
$$

where $t$ is the length of the current pulse and $K$ the slope of the line. The slope depends monotonically on the magnitude of the current pulse used to heat the fiber. The higher this current the faster the temperature increase, and therefore the faster the response.

The linear relationship occurs after the alloy's temperature reaches somewhat past $A_{s}$, the Austenite starting temperature. This effect could be approximated as a time delay, but will not be considered in the closed loop model since while the actuator is under control for normal operation its temperature will be kept in the vicinity of $A_{s}$.

The oscillatory behavior superimposed on the response is due to the linear under-damped second-order dynamics of the system accounting for load inertia, fiber elasticity, and actuator damping. In the controller design, these effects have also been ignored since the system will be designed to track position and consequently will stiffen by a large factor. Under the simple controllers about to be described, all ringing in the response is eliminated for a large range of the load inertial parameters.

Ignoring the time delay, the higher-order response, and the various distortions, our open-loop model is represented by the trajectory curves shown in Fig. 7, which are the response curves of an ideal integrator.

However, the actual open loop response does not match the ideal integrator in that the position is not held when the current is turned off. The mechanical bias due to the preloading springs in the actuator tend to move the set point back slowly toward the neutral position. This effect can be modeled with a first-order system which is in essence a "leaky" integrator. Therefore, using a first-order plant and representing the current-to-temperature relationship also as a first-order, we get the open-loop model shown in Fig. 8. The input to the model is current with the output given by displacement.

To further simplify analysis, the conversion from current into temperature can be replaced by a gain due to the short time constant involved in heating and cooling the 100 micron fibers in the actuator.

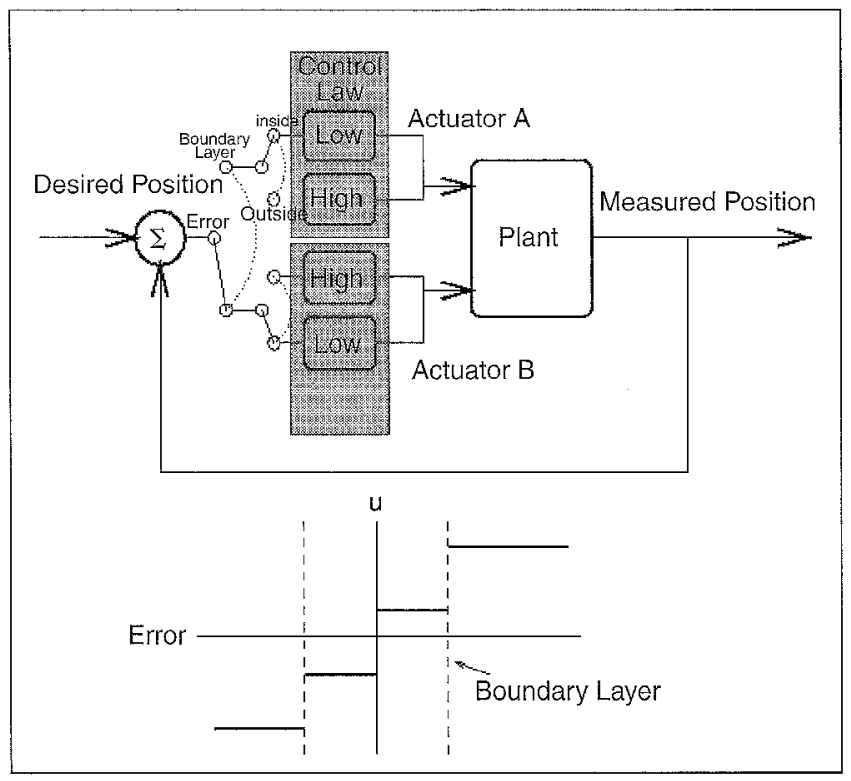

Fig. 11. Block diagram.

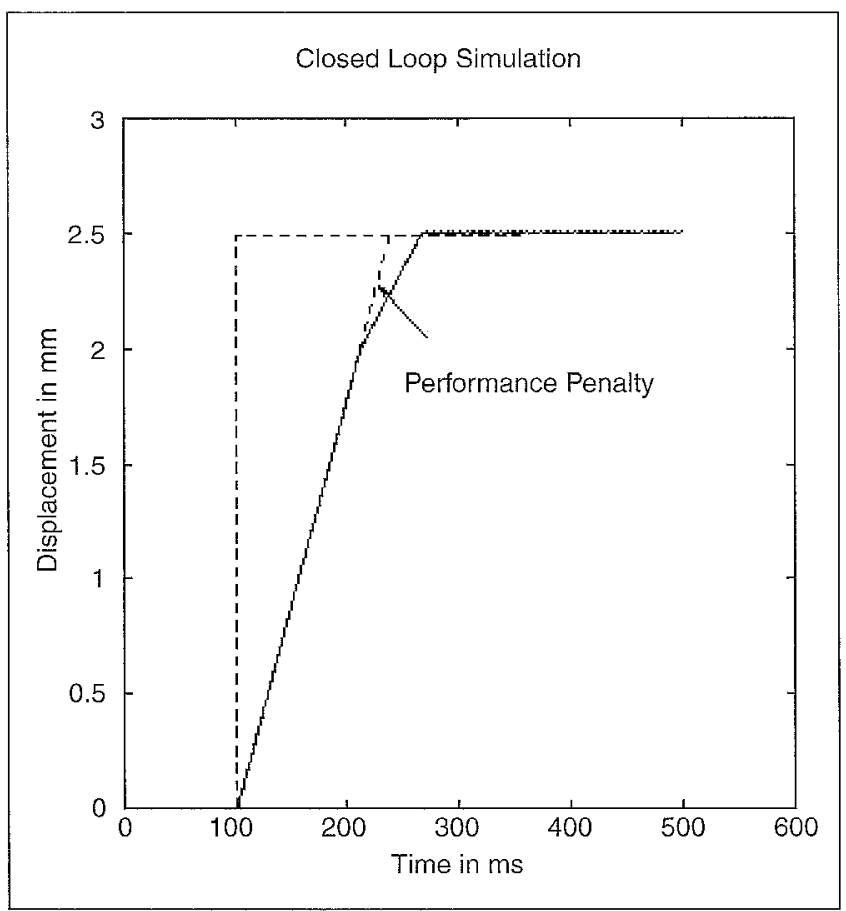

Fig. 12. Model step response.

The chemical energy generated by the phase change is also represented by a linear gain. Depending on the input current magnitude, and hence the temperature of the alloy, we have a corresponding rate of change in position as observed in the openloop curves. This rate can be given by:

$$
\text { rate }=K * \frac{T-A_{s}}{A_{f}-A_{s}} \text { for } A_{s}<T<A_{f}
$$

where $A_{s}$ and $A_{f}$ are the Austenite starting and finishing temperatures. The plant then integrates this velocity to obtain the displacement. 

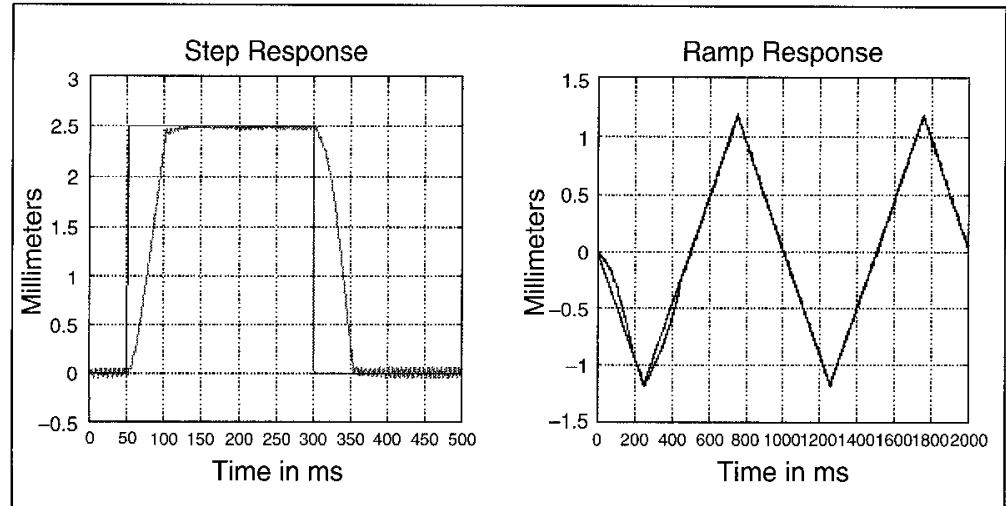

Fig. 13. Step and ramp response of two-stage, constant magnitude.

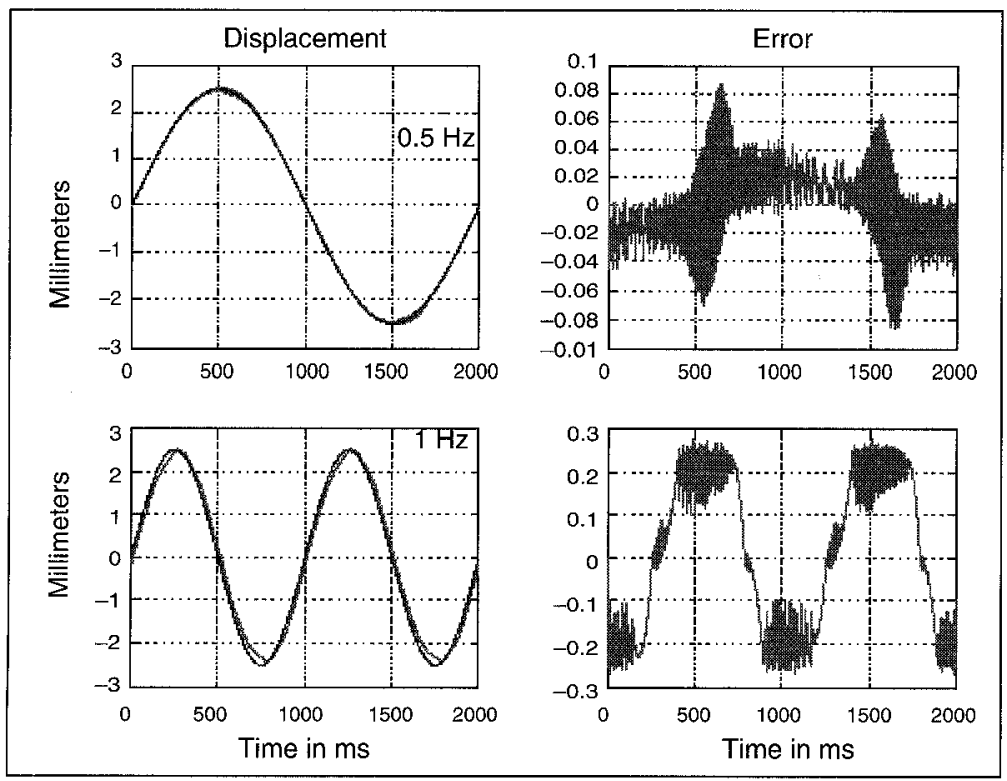

Fig. 14. Sine response of two-stage, constant magnitude.

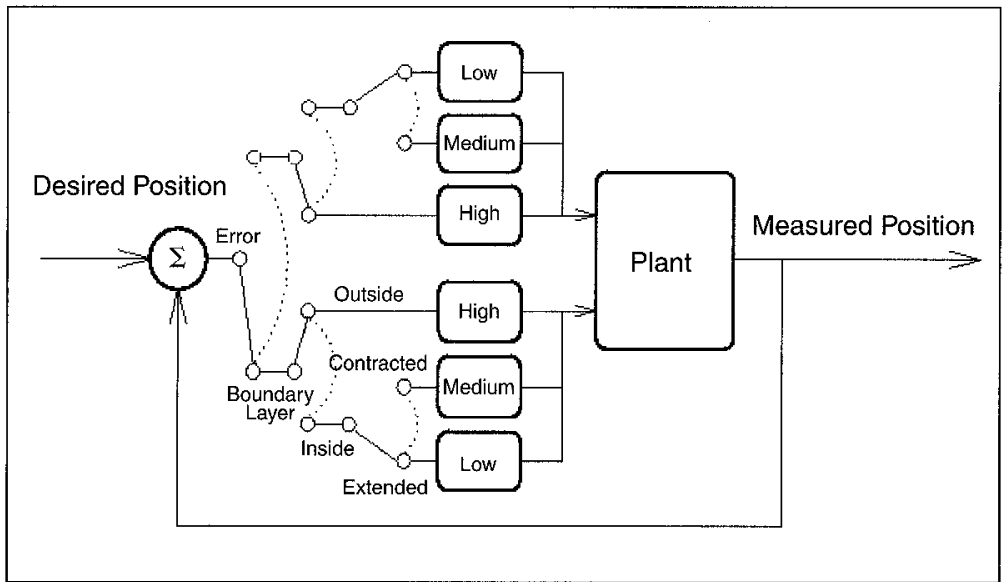

Fig. 15. Block diagram of refined controller.

Choosing a nominal open loop response for a current pulsewidth of $50 \mathrm{~ms}$ at $7.5 \mathrm{amps}$, experimental and simulated results are compared in Fig. 9, where it can be seen that the simple model captures the global flavor of the response while ignoring the local nonlinear effects.
Modeling of Closed-Loop Antagonistic Response

When the antagonist actuators are operating in closed loop, there is always one driving actuator and one passive actuator. Since the force generated by the phase change is much greater than the force required to deform the antagonist actuator, the response is dominated by the agonist actuator. From observing the open-loop curves, it can be seen that while an actuator is being heated the response resembles that of an integrator. Therefore, for closedloop analysis, where one actuator is always driving the system and providing that cooling is sufficiently fast, the model can be further reduced to that of an integrator. The time delay present in the open-loop curves can also be safely neglected since during continuous operation the alloy's temperature will remain around $A_{s}$.

\section{Variable Structure Control Design}

A switching control law makes it possible to drive the state trajectory of a nonlinear plant along a userchosen surface in the state space. Such a surface is referred to as a switching surface since the control gain switches depending on whether the state trajectory is above or below the chosen surface.

The simplest switching surface involves changing the feedback control according to the sign of the error. In the phase plot of error this corresponds to a vertical switching surface along the y-axis as shown in Fig. 10.

Starting from any initial conditions, the state trajectory is driven towards the switching surface, $s$. This is a result of constructing the control gains such that the gradient of the state space vector is always directed toward the switching surface.

With properly chosen feedback gains, the state trajectory can be constrained to move along the switching surface after the initial intersection with the switching surface. Such a motion along aswitching surface is referred to as a "sliding mode," and exists on a discontinuity surface whenever the distance to this surface and the velocity of its change are of opposite signs [14]. More specifically,

$$
\lim _{s \rightarrow 0^{-}} \dot{s}>0 \text { and } \lim _{s \rightarrow 0^{+}} \dot{s}<0
$$

where $s$ is defined as the switching surface.

Given that our model is an integrator, and choosing $s$, the switching surface to be equal to the displacement error $(s=e)$ we have:

$$
x=x_{0}+\int_{t_{o}}^{t} K * i(t) d t
$$

$$
\dot{x}=\dot{e}=K * u
$$

where $x$ is the displacement, $i=u$ is the input current, and $K$ is the actuator gain. The equivalent control, $u_{e q}$, the control needed to stay on the switching surface is then simply given by: 


$$
\begin{gathered}
\dot{s}=\dot{e}=K^{*} u=0 \\
u_{e q}=0
\end{gathered}
$$

The value of $u_{e q}=0$ makes sense due to the fact that our model is an integrator. Once an integrator has a value, it will hold this value if the input is zero. To insure that the trajectories are driven to the switching surface we need to insure $s$ and $s$ are of opposite sign, that is $\dot{s}^{*} s<0$ and so:

$$
\operatorname{sgn}(u)=-\operatorname{sgn}(s) \Leftrightarrow \operatorname{sgn}(u)=\operatorname{sgn}(\dot{s})
$$

In the case of the antagonistic SMA actuator arrangement, this merely entails switching the proper actuator on or off according to the sign of the error. Also note that the selection of which actuator is on and which is antagonistic comes "free" in the nonlinear control design, whereas with a linear controller further adjustments would be required.

Any perturbation from the switching surface results in an immediate control signal that forces the trajectory back on to the switching surface. Infinitely fast switching is required in order for the state trajectory to exactly follow the discontinuity surface. Since actual systems must switch at a finite frequency due to various imperfections, such as hysteresis or delay, the system will enter a limit cycle around the desired point in the phase plane. If a limit cycle is not desired, it is possible to consider a boundary layer around the discontinuity surface, inside which plant is driven smoothly to the surface at the expense of time domain performance. As the imperfections are removed, this boundary layer reduces to the ideal discontinuity surface.

In the time optimal sense, switching is the most efficient way to drive the plant since the maximum realizable gain is used at all times. The major drawback of using maximum drive for highspeed switching is stability. For small perturbations, the maximum feedback gain may cause the system to oscillate or go unstable. The gain must be chosen so that it is large enough to drive the plant as quickly as required to the set point, yet be small enough so as not to cause oscillation larger than a specified limit.

Using a single gain in the feedback path results in a controller that is too conservative, as the desired properties of stability and performance cannot be adequately realized. Hybrid switching controllers have also been proposed [4], notably a two-stage switching control law. In the two-stage approach different feedback gains are used as one approaches the discontinuity surface. This results in a controller with several switching surfaces; however, the surface defined by the set point is the only surface to exhibit sliding by the choice of the feedback gains.

\section{Two-Stage Controller}

A two-stage hybrid controller has been explored for the control of the SMA actuator. Two constant amplitude current values are used depending on the magnitude of the error. When the error is large, a high value of current is used to drive the plant quickly to the set point. As the error approaches zero, a lower magnitude pulse is used to minimize the magnitude of the limit cycle. This results in a smoother motion as the state trajectory slows down as it approaches the set point switching surface, allowing us to satisfy the dual requirements of having stability and a quick response.

The adjustable parameters for the two-stage controller are the location of the threshold switching surfaces and the amplitudes of the constant current high and low pulses. A good value for this gain near the set point is the current level needed to maintain the temperature of the actuator, that is, a current that will provide enough heat to compensate for the ambient heat loss.

The block diagram of the controller can be seen in Fig. 11.

\section{Applying the Controller}

\section{On the Model}

Fig. 12 shows the step response when applying the two-stage feedback controller on the model previously discussed. Recall that the model in closed loop simplifies to an integrator with a gain. The boundary layer is set at $.5 \mathrm{~mm}$ with a high pulse of 6.0 amps and a low pulse of 3.0 amps.

The response clearly shows the transition from the high to low pulse at the threshold boundary level. The boundary layer should be made as small as possible, dictated by stability constraints, as better time performance will result.

\section{On the Actuator}

Fig. 13 shows the step and ramp response of the two-stage constant controller with the parameters set as follows: (1) maximum gain = $6.3 \mathrm{amps}$; (2) gain near set point = 3.4 amps; (3) threshold level $=.188 \mathrm{~mm}$.

The step response here enters a limit cycle of $160 \mathrm{~Hz}$ with an average value steady state error of $.01 \mathrm{~mm}$. The rise time is 35 $\mathrm{ms}$. The limit cycle at the set point has a magnitude of $.04 \mathrm{~mm}$, while the limit cycle near the neutral position has a magnitude of $.1 \mathrm{~mm}$. The tracking response for a $1 \mathrm{~Hz}$ triangle wave is quite accurate after the initial half wavelength. This is due to the fact that the actuators initially begin at room temperature and there is a time delay when heating the cold actuators. During continuous operation, however, the actuator's temperature remains near $A_{s}$ and the tracking is accurate. This experimentally justifies the neglection of a time delay in the proposed closed-loop model. Fig. 14 shows the response of two different sinusoidal frequencies. While the plant is able to reasonable track a sine wave of $.5 \mathrm{~Hz}$, it lags behind a sine wave of $1 \mathrm{~Hz}$. This lag is a result of having too low of a gain within the boundary layer; however, this low-gain is necessary for stability requirements.

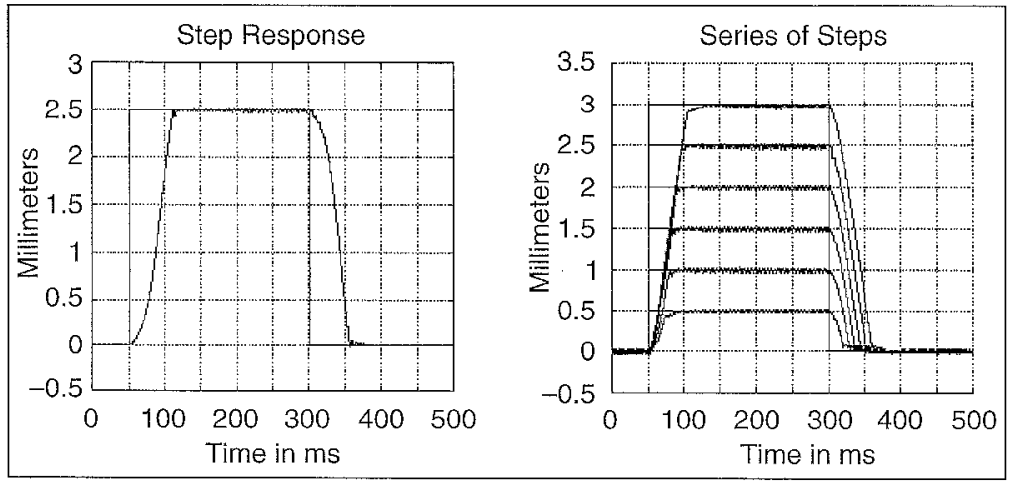

Fig. 16. Step response of refined controller. 
In examining the step response it can be seen that the limit cycle possesses a different magnitude at the $2.5 \mathrm{~mm}$ mark compared to the neutral position. This is a by-product of attempting to compensate for the nonlinear displacement gain of the actuator with one fixed magnitude pulse. When the actuator is extended, it has a different displacement gain than when it is contracted. As a refinement to the two-stage constant-magnitude controller, a third constant-magnitude pulse was added to the controller depending on the state space information of whether the actuator was extended or contracted. This will allow us better control over the limit cycle magnitude throughout the working range of the actuator. The switching scheme is then given as shown in Fig. 15, and a step response is shown in Fig. 16.

Comparing the left side of Fig. 16 with Fig. 13 we can see that the limit cycle is indeed reduced around the neutral position.

Ideally, the constant-magnitude pulse inside the boundary layer should have an amplitude that compensates for the displacement force tradeoff throughout the workspace of the actuator. However, the right side of Fig. 16 indicates that this added complexity is perhaps unnecessary.

\section{Disturbance Rejection}

By construction of the set point switching surface, any deviance from the set point results in an immediate gain that drives the plant back to the set point surface. In Fig. 17 a disturbance force of $2 \mathrm{~N}$ is applied by the testbed at $100 \mathrm{~ms}$. This corresponds to $50 \%$ of the actuator's maximum rated force.

\section{Observations}

Shape memory alloys, however, are notorious for numerous nonlinearities, including dominating hysteresis loops. Why, then, does such a simple model, an integrator, produce accurate results? We have found that the antagonist arrangement chosen for the actuators, combined with the method of control, was the cause of the complexity reduction. While under the influence of control, there is at all times only one actuator driving the system. That is, one actuator undergoing transition from the Martensite phase to the Austenite phase, while the other remains mostly in the malleable Martensite phase. The result of this is that the drive is always derived from the same side of the hysteresis loop, be it a major or a minor loop. As an analogy to this consider the double gear systems in anti-backlash drives. Moreover, the return path of the passive actuator acts as a constant bias that will allow the driving actuator to be firmly in control, regardless of the sign of an exogenous disturbance. These combined effects tend to cancel out the hysteresis, however not perfectly; thus the robustness properties of switching control are then exploited to compensate for the remaining imperfections.

\section{Conclusions}

A novel actuator using shape memory alloys has been proposed that overcomes the main drawback of shape memory alloys, that being limited strain. The abundant force available with SMA fibers is efficiently transformed to increase displacement by weaving fibers around supporting disks. An actuator prototype (see Fig. 18) has been constructed with the following properties:

- light weight-6 grams

- compact-17 mm cylinder x $30 \mathrm{~mm}$ long

- powerful-4 newtons

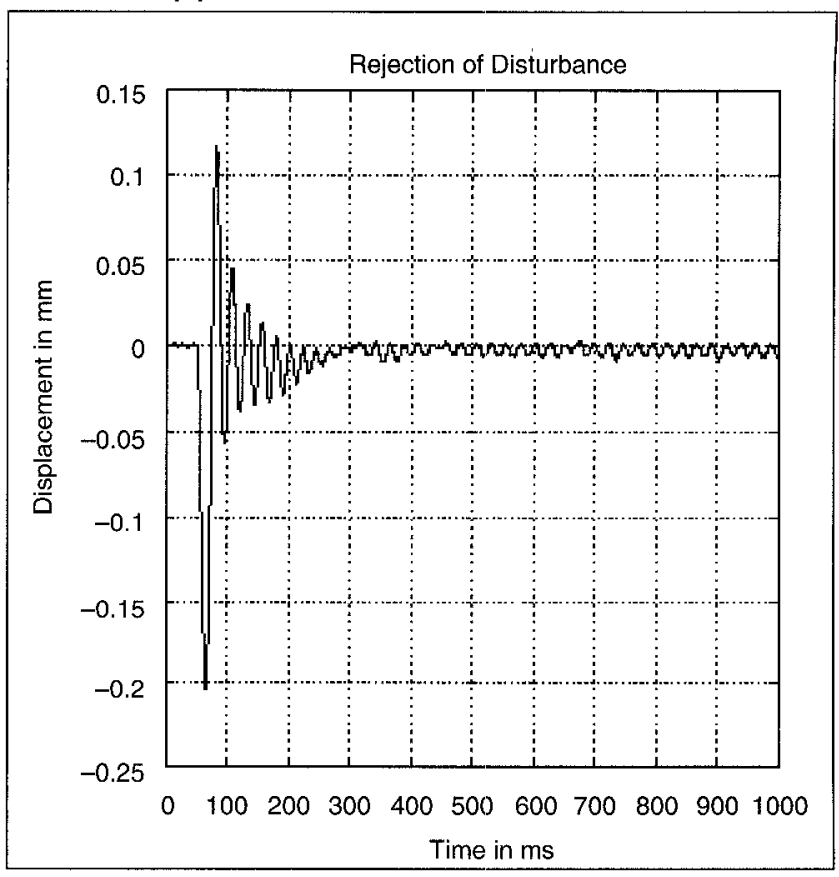

Fig. 17. Disturbance rejection with refined controller:

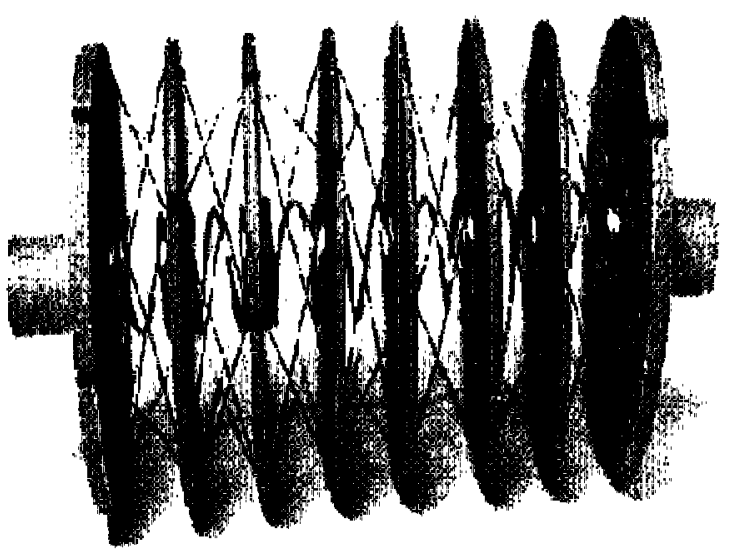

Fig. 18. SMA actuator prototype.

- direct drive actuator

- requires no gears or lubrication

- smooth movements

- silent

- modular

Open loop experiments were conducted to demonstrate the intrinsic properties of the SMA actuator. Using these graphs and knowledge of the underlying physics, a simple model was purposed and further justified through simulation. Two multilevel switching control laws were then applied to the shape memory alloy actuator. The controllers are based on a simple concept, switching on the sign of the error, and produces rather satisfactory results. Further research has been done on implementing this technology for a compact pan, tilt, and torsion camera unit [5]. 


\section{IEEE Systems and Control Maganize, Vol. 17, No. 3, pp. 80-88, 1997}

\section{Acknowledgments}

The research was mostly funded by the Institute for Robotics and Intelligent Systems (IRIS), part of Canada's National Centers of Excellence program (NCE). Additional funding was provided by a team grant from le Fond pour les Chercheurs et l'Aide a la Recherche (FCAR), Quebec, and an operating grant from the National Science and Engineering Council of Canada (NSERC). The support of Prof. Martin D. Levine was essential to the completion of this project.

\section{References}

[1] T.W Duerig, K.N. Melton, D. Stockel, and C.M. Wayman, Engineering Aspects of Shape Memory Alloys. Butterworth-Heinemann Ltd., Toronto, 1990.

[2] D. Yang, G. Lin, and R.Warrington, "A Computational Model of Shape Memory Alloys for the Design and Control of Micro-Actuators," Micromechanical Systems, vol. 40, pp. 171-181, 1992.

[3] Buckminster Fuller. Inventions: The Patented Work of Buckminster Fuller, St. Marlin's Press, Toronto, 1983.

[4] John E. Gibson, Nonlinear Automatic Control, McGraw-Hill Ine., 1963.

[5] D. Grant, "Shape Memory Alloy Actuator with an Application to a R. botic Eye," master's thesis, McGill University, 1995.

[6] M. Hashimoto, Mansanori Takeda, Hirofumi Sagawa, and Ichiro Chiba, "Application of Shape Memory Alloy to Robotic Actuators," Joumal of Robotic Systems. 2(1):3-25, 1985.

[7] I. Hunter. S. Lafontaine, J. Hollerbach, and P. Hunter, Fast Reversible NiTi Fibers for Use in Microrobotics. In Microelectro-Mechanical Systems, pp. 166-170, Nara, Japan, January 1991.

[8] K. Ikuta, "Micro/Miniature Shape Memory Alloy Actuator," in IEEE Robotics and Automation, vol. 3, pp. 2156-2161, Los Alamitos, CA, May 1990. IEEE Computer Society Press.

[9] K. Ikuta, M. Tsukamoto, and S. Hirose. "Mathematical Model and Experimental Verification of Shape Memory Alloy for Designing Micro Actuator," in Proc. of the IEEE MicroElectroMechanical Systems Conference, pp. 103-108, 1991.
[10] K. Kuribayashi, "A New Actuator of a Joint Mechanism Using TiNi Alloy Wire." Int. Journal of Robotics Research, 4(4), pp. 47-58, 1986.

[11] W. Li and J-J. E. Slotine, Applied Non-Linear Control, Prentice Hall, 1991.

[12] D.R. Madill, "Modeling and Stability of a Shape Memory Alloy Position Control System," master's thesis, Applied Science, University of Waterloo, 1993.

[13] D. Reynaerts and H. Van Brussel. "Development of a SMA HighPerformance Robotic Actuator," in Fifth International Conference on Advanced Robotics, vol. 2, pp. 19-27, New York, NY, 1991.

[14] V.L. Utkin, Sliding Modes in Control Optimization, Springer-Verlag, 1992.

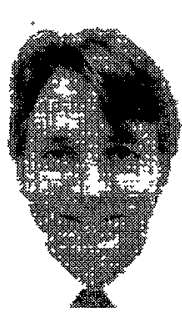

Danny Grant was born in Edmonton, Alberta, on October 10, 1968. After completing his bachelor of electrical engineering in 1992, at McGill University, he undertook a master's degree, for which he developed a high-speed prototype camera mount using shape memory alloy actuators. He currently is fulfilling his doctoral requirements in the area of nonlinear control with applications to robotics at the Center for Intelligent Machines at McGill University.

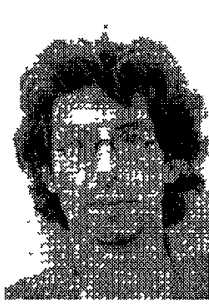

Vincent Hayward received the Diplome d'Ingenieur from Ecole Nationale Superieure de Mecanique de Nantes, Nantes, France, and the Diplome de DocteurIngenieur from Universite de Paris XI at Orsay in computer science, in 1978 and 1981, respectively. He held research positions at Purdue University, and at CNRS, France. He is currently associate professor with the Department of Electrical Engineering at McGill University and a member of the Center for Intelligence at McGill. His contributions include RCCL and Kali, robot control and programming systems now widely used in many application areas. His research interests are in the following areas: robotics, actuators, mechanisms, and haptic devices. He is the leader of the project "Haptic Devices for Teleoperation and Virtual Environments" of the Institute for Robotics and Intelligent Systems (IRIS), one of Canada's network of Centers of Excellence. He is a member of the IEEE and presently an associate editor of the IEEE Transactions on Robotics and Automation. 